\title{
Teledermatology for all? A service evaluation of mandatory teledermatology in Cardiff and Vale UHB 2016-17
}

\author{
Authors: Nutchanun Poolworaluk ${ }^{A}$ and Richard Motley ${ }^{B}$
}

\begin{abstract}
Introduction
With over 10 years of experience in providing a teledermatology service, the department of dermatology in the University Hospital of Wales now requires all general practitioner (GP) referrals to be in the form of teledermatology. Referrals are reviewed by a dermatologist within 2-3 days and may be accepted or returned with diagnosis and treatment advice. ${ }^{1}$ Dermatology is a topic that is taught less extensively in medical school and only a handful of GPs received dermatology training, yet around $20 \%$ of GP appointments every year concern patients with skin conditions. ${ }^{2}$ This results in long waiting lists for dermatology clinics and unnecessary outpatient appointments for conditions that, given the correct advice from specialists, could be actively managed by their GP. ${ }^{3}$ This project evaluated the teledermatology service after its first year and sought opinions from the key user groups (GPS, consultants, patients and office administrators) to identify any concerns, risks or deficiencies in the current service and potential opportunities for its future improvement.
\end{abstract}

\section{Materials and methods}

Details about the workflow and opinions about the service were recorded for each group. Thirty general practices and all nine dermatology consultants in the Cardiff and Vale University Health Board were sent an online questionnaire. Office administrators and patients were interviewed in person for their opinions. Statistics on the service were collated and the metrics of the service were analysed for variation between dermatology consultants. Two studies were undertaken: Study 1, intraconsultant variation - to analyse consistency of diagnosis within individual consultants; and Study 2, interconsultant variation - to analyse differences in diagnostic opinion between consultants.

\section{Results and discussion}

From September 2016 to September 2017, 17,467 patient cases were sent to dermatologists via teledermatology; $26 \%$ were returned by the consultant, $3 \%$ were redirected to other specialties, and the rest were accepted into dermatology clinics. Thirty-eight per cent of the referred cases were urgent or urgent suspected cancer cases while $62 \%$ of those were routine cases. Results from users showed appreciation of this new service and few problems such as technical difficulty were highlighted. Intraconsultant variation study showed $79 \%$ consistency in diagnosis over a 6-month interval, where the diagnosis of the inconsistent cases was uprated. Interconsultant variation study showed a wide variation of acceptance of routine cases but unanimity in urgent cases.

\section{Conclusion}

Given the results shown, it is safe to say that teledermatology is here to stay. The service is appreciated by both the patients and doctors. The removal of unnecessary appointments in conjunction with 'fast track' appointments for urgent cases are highly valued by patients. GPs think highly of the advice given by the consultants, which has allowed the practitioners to advance their primary care and professional development. Consultants found the service excellent for triage and management of increased need for dermatology appointments.

\section{Conflicts of interest}

None declared.

\section{References}

1 Eedy DJ, Wootton R. Teledermatology: A review. Br J Dermatol 2001;144:696-707.

2 Royal College of Physicians. Consultant physicians working with patients. London: RCP, 2013.

3 Edwards N, Imison C. How can dermatology services meet current and future patient needs, while ensuring quality of care is not compromised and access is equitable across the UK? The King's Fund, 2014. www.bad.org.uk/shared/get-file.ashx?id=2348\&itemtype =document [Accessed 22 January 2019].

Authors: ${ }^{A}$ Cardiff University School of Medicine, Cardiff, UK;

${ }^{B}$ Cardiff University, Cardiff, UK 